\title{
Optimization of the Kinematics of a Flapping Wing MAV in Hover for Enhanced Performance
}

\author{
A. Gogulapati* \\ Department of Mechanical 83 Aerospace Engineering, The Ohio State University, Columbus, 43210, USA
}

\author{
P. P. Friedmann ${ }^{\dagger}$ and J. R. R. A. Martins ${ }^{\ddagger}$ \\ Department of Aerospace Engineering, The University of Michigan, Ann Arbor, 48109, USA
}

The kinematics of a rigid and flexible flapping wing micro air vehicle (MAV) in hover are optimized for enhanced performance using surrogate based approximation of the unsteady aerodynamic loads. The surrogates are generated using kriging interpolation of the time averaged thrust generated and power consumed by the wings. The fitting data is obtained using a nonlinear approximate aeroelastic model that combines a nonlinear finite element based structural dynamic model with an approximate unsteady aerodynamic model. The parameter space for the rigid wings consists of seven kinematic parameters. For the flexible wing case, six structural parameters are used to augment the kinematic parameters, resulting in a total of 13 design variables. The surrogates are combined with a hybrid search algorithm to identify feasible designs that produce the desired combination of thrust and power. The best rigid and flexible configurations predicted using the surrogates are superior to the best designs available in the corresponding sets of sample points used to create the surrogates. Thrust and power are found to be competing design objectives. The phase angle between flap and pitch motions has a significant impact on the wing performance for fixed stroke amplitudes and frequency. The peak performance of flexible wings occurs for smaller amounts of pitch actuation compared to the rigid wings due to elastic wing twist. Rigid wings performed better than flexible wings in several regions of the parameter space, leading to the conclusion that the choice between rigid or flexible wings is not straightforward.

\section{Nomenclature}

$\begin{array}{ll}\mathrm{A}_{\mathrm{w}} & \text { Wing area } \\ a & \text { Distance between pitching axis and mid-chord of the airfoil } \\ c_{r} & \text { Root chord } \\ E & \text { Elastic modulus } \\ e & \text { Error } \\ \boldsymbol{F}_{s p} & \text { Force vector in the }\left(X_{S P}, Y_{S P}, Z_{S P}\right) \text { coordinate system } \\ f & \text { Flapping frequency } \\ K_{\Phi} & \text { Shape parameter in flap } \\ K_{\alpha} & \text { Shape parameter in pitch } \\ j, k & \text { Indices } \\ N_{d v} & \text { Number of design variables } \\ N_{\text {sections }} & \text { Total number of aerodynamic span stations } \\ N_{s p} & \text { Number of sample points } \\ N_{t e s t} & \text { Number of test points } \\ N_{\theta} & \text { Discretization of the circle }\end{array}$

* Post Doctoral Research Fellow.

$\dagger$ François-Xavier Bagnoud Professor, Fellow AIAA.

$\ddagger$ Associate Professor. 


\begin{tabular}{|c|c|}
\hline $\begin{array}{l}\hat{\boldsymbol{n}}_{w} \\
n_{w k s u b i t}\end{array}$ & $\begin{array}{l}\text { Instantaneous normal vector at point on wing, resolved in the } X_{S P} Y_{S P} Z_{S P} \text { coordinate system. } \\
\text { Number of wake sub-iterations }\end{array}$ \\
\hline Paero & Aerodynamic power \\
\hline$p$ & Local static pressure on the airfoil \\
\hline$p_{\infty}$ & Free stream pressure \\
\hline$p_{\text {applied }}$ & Applied pressure on the wing \\
\hline$p_{\text {computed }}$ & Pressure computed using the unsteady Bernoulli principle \\
\hline$p_{k}$ & Correlation parameter in kriging \\
\hline$p_{\text {limit }}$ & An upper bound for pressure \\
\hline$p_{\text {ref }}$ & Reference pressure \\
\hline $\mathbf{R}_{k r g}$ & The correlation matrix \\
\hline$R_{j}$ & Radial location of the $j^{t h}$ spanwise section with respect to the wing root \\
\hline$R_{\text {span }}$ & Span of the wing, measured from root to the tip \\
\hline$R_{k r g}$ & Elements of the correlation matrix \\
\hline $\mathbf{r}_{k r g}$ & Correlation matrix computed at a point where surrogate prediction is needed. \\
\hline$r_{c}$ & Vortex core radius \\
\hline$s$ & Mean square error \\
\hline$T$ & Time period \\
\hline$t$ & Time \\
\hline$t_{\text {le spar }}$ & Thickness of leading edge spar \\
\hline$t_{R i b 1}-t_{R i b 5}$ & Thickness of the ribs (battens) \\
\hline$U_{\text {ref }}$ & Reference speed \\
\hline$U_{t i p}$ & Maximum tip speed \\
\hline$w$ & Weight used in the weighted expected improvement function \\
\hline$\left(X_{S P}, Y_{S P}, Z_{S P}\right)$ & Coordinate system fixed to the stroke plane \\
\hline$\left(X_{w}, Y_{w}, Z_{w}\right)$ & Wing fixed coordinate system \\
\hline $\boldsymbol{x}_{w}$ & Instantaneous position vector of a point on the wing in the $\left(X_{S P}, Y_{S P}, Z_{S P}\right)$ coordinate system \\
\hline $\mathbf{x}$ & Vector of design variables \\
\hline$y$ & Function to be approximated \\
\hline $\mathbf{y}$ & Vector of $y$ 's corresponding to all sample points \\
\hline$\hat{y}_{k r g}$ & Kriging prediction of $y$ \\
\hline$Z(\mathbf{x})$ & Deviation about the global mean, assumed to be a stochastic process \\
\hline \multicolumn{2}{|l|}{ Greek Symbols } \\
\hline $\bar{\alpha}$ & Pitch angle \\
\hline$\alpha_{1}$ & Pitch amplitude \\
\hline$\beta$ & Constant \\
\hline$\hat{\beta}$ & Least-squares approximation of $\beta$ \\
\hline$\Phi$ & Flap angle \\
\hline$\Phi_{1}$ & Flap amplitude \\
\hline$\Phi_{\text {dist }}$ & Standard normal distribution function \\
\hline$\phi_{d e n}$ & Standard normal density function \\
\hline$\varphi_{\alpha}$ & Phase angle between flap and pitch motions \\
\hline$\vartheta_{k}$ & Correlation parameter in kriging \\
\hline$\sigma_{v a r}$ & Variance \\
\hline$\hat{\sigma}_{\text {var }}$ & Least-squares approximation of the variance \\
\hline$\nu_{\infty}$ & Kinematic viscosity of the fluid \\
\hline$\nu$ & Poisson's ratio \\
\hline$\rho$ & Density of the material \\
\hline$\rho_{\infty}$ & Free stream density of the fluid \\
\hline \multicolumn{2}{|c|}{ Additional Subscripts and Superscripts } \\
\hline$|\cdot|$ & Absolute value or magnitude \\
\hline$(\dot{*})$ & Derivative with respect to time \\
\hline
\end{tabular}




\section{Introduction}

During the last fifteen years, interest in micro air vehicles (MAVs) for both military and civilian applications involving confined spaces, such as buildings, or short distances has been growing. Typical dimensions of such vehicles are less than $15 \mathrm{~cm}$ and their maximum weight is less than 100 grams. The MAVs are expected to operate at low Reynolds numbers $\left(10^{2}<R e<10^{4}\right)$ and low speeds in forward flight $(<15 \mathrm{~m} / \mathrm{s}) .{ }^{1}$ Bio-inspired flapping wing designs of hover-capable biological flyers, such as insects and hummingbirds, are of interest due to their exceptional flight capabilities. ${ }^{1}$

The wing kinematics of hover capable biological and bioinspired flyers consists predominantly of flapping and pitching motions, combined with a comparatively small elevation motion. ${ }^{1}$ Research on the aerodynamics of flapping wings ${ }^{1-6}$ indicates that the forces generated by the wings can vary significantly in the design space described by the kinematic parameters. Furthermore, the power available for use for a practical MAV is expected to be limited due to the restrictions on size and weight. ${ }^{7}$ Therefore, the selection of kinematics that produces the 'best' combination of thrust and power is critical to the development of practical MAVs.

Optimization of the kinematics of rigid and flexible wings was considered in Refs. 8-10. In [8], a semi-empirical aerodynamic model was combined with a global optimization algorithm to identify powerminimizing kinematics for several rigid bio-inspired wing configurations in hover, actuated by a combined flap-pitch motion. Optimal kinematics were found to be similar to the kinematics observed in the corresponding biological flyers. The lift and propulsive efficiency (lift generated per unit power) of rigid airfoils, computed using CFD, and undergoing combined pitch-plunge motions in a free stream were maximized using a gradient-based (GB) optimization algorithm. ${ }^{9}$ Kinematics producing leading edge vortices (LEVs) increased the lift and decreased the propulsive efficiency. Kinematics that prevented the formation of LEVs maximized the propulsive efficiency. In Ref. 10, optimal combinations of wing kinematics, flexibility, and planform were identified using a GB optimization approach. The wings, modeled as beams, were actuated by a combined flap-pitch-elevation motion. The aerodynamic loads and power were computed using a quasisteady formulation. Wing flexibility and shape were found to be significant for reducing the power generated by the wings.

In the current paper, the aerodynamic force and power of the MAV wings are calculated using a nonlinear approximate aeroelastic model ${ }^{11,12}$ that shows acceptable correlation with CFD simulations and experiments for a wide range of kinematic, structural, and aerodynamic parameters. The model offers a significant reduction in computational cost compared to CFD based approaches. However, the cost for the large number of simulations required in an optimization study prevents the direct coupling of the aeroelastic analysis with the optimization algorithm. Therefore, surrogate models, which represent computationally efficient global approximations of the analysis, ${ }^{13}$ are used.

The surrogates are constructed by interpolating the input/output data obtained from a suitable number of true function evaluations and are used to replace the expensive computations when exploring the parameter space. ${ }^{13}$ In previous flapping wing studies ${ }^{14}$ surrogate approaches have been limited to rigid wings. Surrogates based on CFD data were used to investigate the impact of LEVs and tip vortices on the lift and thrust generated by rectangular wings undergoing prescribed translation and pitching motions. The surrogates, constructed by interpolating the time averaged forces generated by the wing as a function of the amplitudes and phase difference of wing motions, were used to identify regions of the parameter space where tip vortices had a beneficial effect.

The overall goal of the paper is to identify combinations of wing kinematics and flexibility that produce improved performance, as measured by thrust and power requirement, for anisotropic flapping wings in hover. The specific objectives of this paper are to:

1. Develop accurate surrogate models that predict the performance of rigid and anisotropic flapping wings in hover.

2. Identify optimal combinations of wing kinematics and flexibility to achieve the desired performance and satisfy the specified constraints.

Achieving these objectives represents an important advance in the ability to design practical MAV configurations. 


\section{Nonlinear Aeroelastic Model}

The aeroelastic model ${ }^{11,12}$ is obtained by coupling a nonlinear finite element model of the wing with an approximate aerodynamic model that incorporates LEV's and a free wake. The ingredients of the model are concisely described next.

\section{A. Structural Dynamic Model and Wing Kinematics}

The structural dynamic model is based on MSC MARC, ${ }^{15}$ a commercially available nonlinear finite element code. Shell elements capable of modeling large rigid body rotations as well as moderate-to-large flexible deformation are used. The structural dynamic model is suitable for modeling both isotropic and anisotropic wings. ${ }^{15}$ Wing kinematics, which consist of large amplitude rigid body rotations prescribed at the root of the wing, are applied as displacement boundary conditions at two or more nodes. Additional details on the model can be found in Ref. 16 (Chapter 2).

\section{B. Approximate Aerodynamic Model}

The unsteady aerodynamic formulation ${ }^{11,12}$ is based on a modified version of the model originally described in $[17,18]$. The modifications introduced account for wing flexibility, forward flight and fluid viscosity. The formulation is based on 2D potential flow and uses a vorticity/circulation approach to compute the aerodynamic loads. The presence of LEV is assumed, i.e. it is either present or not, along with the location of the separation point.

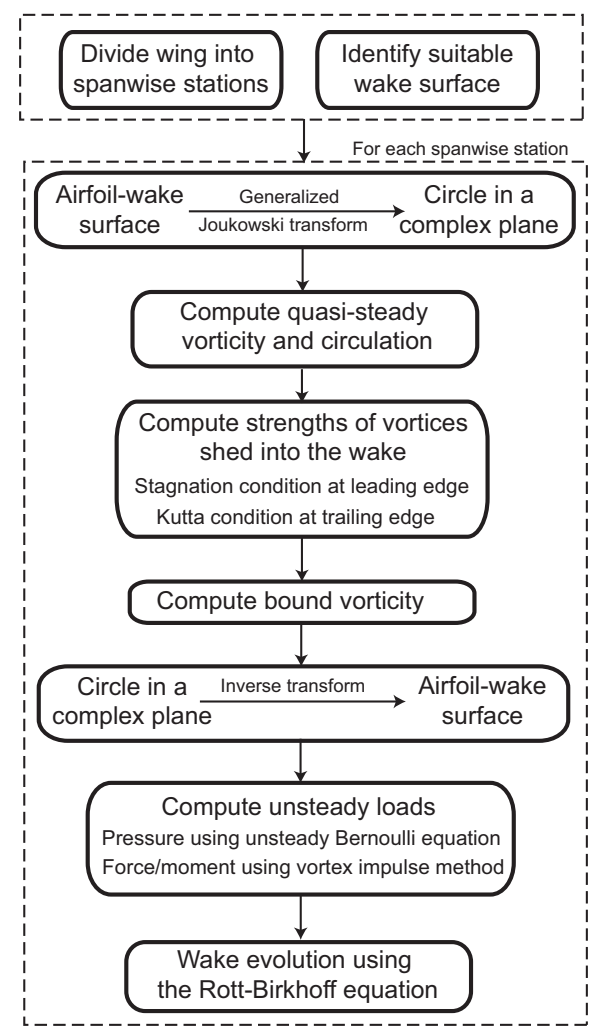

Figure 1. Schematic of the aerodynamic model

The overall approach is summarized in Fig. 1. First, the wing is divided into several spanwise stations, shown in Fig. 2(a), where each section is represented as an airfoil. For each airfoil, the airfoil motion and the shed wake geometry are approximated using an airfoil-wake surface that is represented by a cylinder normal to the stroke plane (SP), shown in Fig. 2(b). The cylindrical surface is straightened out (unwrapped) to represent a $2 \mathrm{D}$ plane. The airfoil and shed vortices are modeled in this plane by transforming the airfoil into a circle on a complex plane using a conformal mapping. The bound and shed wake vorticity are computed 
on the complex plane. The quasi-steady component of vorticity is obtained by neglecting the effect of the shed wake. The strength of shed vorticity is computed by enforcing a stagnation condition at the leading edge (LE) and a Kutta condition at the trailing edge (TE). The airfoil bound vorticity is obtained as a sum of the quasi-steady and wake-induced vorticity on the airfoil. Next, the vorticity on the complex plane is transformed back to the airfoil-wake surface using an inverse transform. Subsequently, the unsteady aerodynamic pressure is computed from the total vorticity using the unsteady Bernoulli equation. Finally, the shed vorticity is convected using the Rott-Birkhoff equation, derived from Biot-Savart law for 2D flow. The modified formulation and its implementation are described in Refs. 11 and 12.

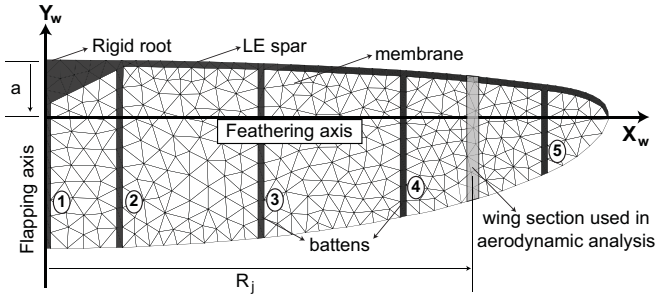

(a)

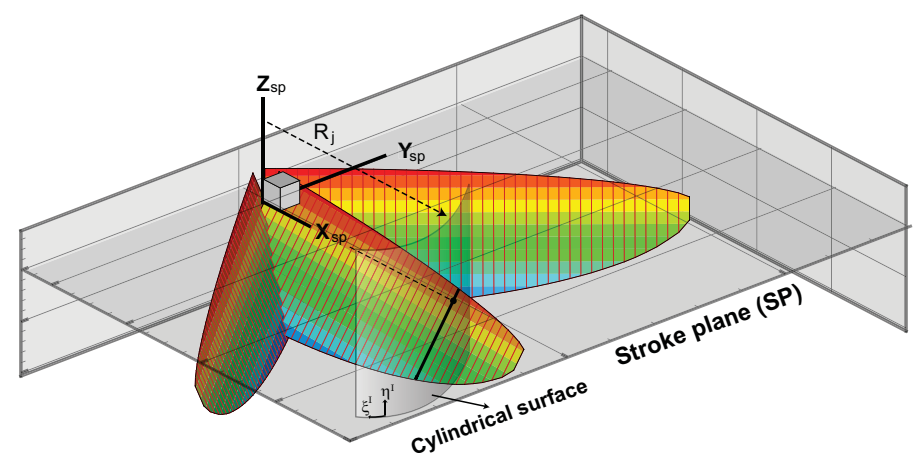

(b)

Figure 2. Stroke plane and normal cylinder

\section{Fluid-structure Coupling}

The fluid-structure coupling between the approximate aerodynamic model and the structural dynamic model represented by MARC is accomplished by two user-defined subroutines in MARC, FORCDT and FORCEM. The FORCDT subroutine allows the user to specify displacement and/or rotation boundary conditions at selected nodes and is used to prescribe wing kinematics at the root. The FORCEM subroutine allows the user to specify distributed loads on selected elements and is used to apply the aerodynamic loads on the structure.

The equations of motion (EOM) representing the aeroelastic problem are obtained in MARC using an updated Lagrangian (UL) method, Ref. 19 (Ch. 6). The implementation of the aeroelastic model is illustrated in the block diagram shown in Fig. 3. At each time step, rigid body motion is prescribed as displacements at specified nodes using the FORCDT subroutine. The aerodynamic loads, computed based on the wing motion at the beginning of each time step, are applied as pressure on the structure using the FORCEM subroutine. This subroutine is called from the main program for each step of the Newton-Raphson iteration within a time step to ensure convergence of the structural displacements for the applied loads. Finally, the vortices shed into the wake are convected at the end of the time step. Additional details are provided in Ref. 16 (Chapter 4). 


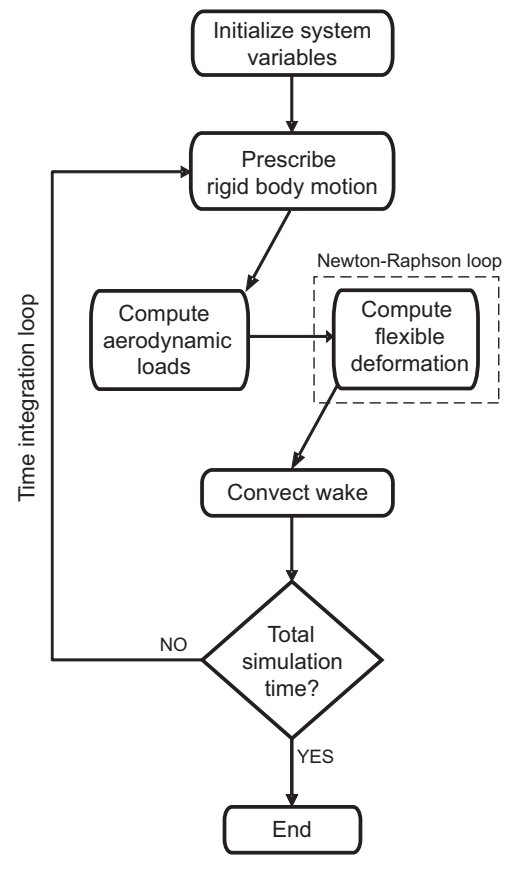

Figure 3. Flowchart for the aeroelastic model in MARC

\section{Surrogate Modeling and Optimization}

Surrogates are used to predict the time averaged aerodynamic force and power as functions of the kinematic and structural parameters. The surrogate modeling approach followed here is similar to that described in Ref. 20, and is illustrated in Fig. 4. The various steps are summarized next.

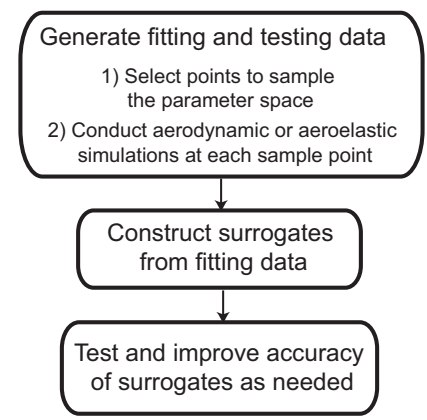

Figure 4. Steps in surrogate modeling

\section{A. Generation of the Surrogate and Testing Its Accuracy}

Initially the surrogates are constructed using a set of fitting points selected to sample the parameter space in an efficient manner. These points are generated using an optimal Latin hypercube (OLH) sampling. ${ }^{13}$ In this approach, the design variables are partitioned into several equally spaced sections, thereby dividing the parameter space into several equally sized hypercubes. A spreading criterion is used ensure uniform sampling in the design space. The accuracy of the surrogates is estimated at a set of testing points that are not included in the set of fitting points. The testing points are also generated using an OLH sampling in order to represent the parameter space in an efficient manner.

The aerodynamic or aeroelastic simulations are performed at each of the design points. The simulations are run in parallel on several processors since the true function evaluation at each design point can be run independently of the evaluations at other design points. 
The surrogates are created from the fitting data using kriging interpolation following the approach used in Ref. 20. In kriging, the unknown function, denoted by $y(\mathbf{x})$, is approximated by:

$$
y(\mathbf{x})=f(\mathbf{x})+Z(\mathbf{x})
$$

Where, $f(\mathbf{x})$ is the global mean and $Z(\mathbf{x})$ is the deviation about the global mean, represented as a stochastic process. The covariance matrix of $Z(\mathbf{x})$ (Ref. 21, Ch. 2), which is a measure of how strongly correlated two points are, is given by

$$
\operatorname{Cov}\left[Z\left(\mathbf{x}^{(i)}\right), Z\left(\mathbf{x}^{(j)}\right)\right]=\sigma_{v a r}^{2} \mathbf{R}_{k r g}
$$

where each element of the correlation matrix $\mathbf{R}_{k r g}$ is given by

$$
\left(R_{k r g}\right)_{i j}=R_{k r g}\left(\mathbf{x}^{(i)}, \mathbf{x}^{(j)}\right)
$$

The function $R_{k r g}\left(\mathbf{x}^{(i)}, \mathbf{x}^{(j)}\right)$, called the spatial correlation function (SCF), is user specified and accounts for the effect of each interpolation point on every other point. A commonly used form of the SCF is the generalized Gaussian function: ${ }^{21}$

$$
R_{k r g}\left(\mathbf{x}^{(i)}, \mathbf{x}^{(j)}\right)=\exp \left[\sum_{k=1}^{N_{d v}} \vartheta_{k}\left|\mathbf{x}_{k}^{(i)}-\mathbf{x}_{k}^{(j)}\right|^{p_{k}}\right]
$$

where, $\vartheta_{k}$ and $p_{k}$ are correlation parameters that determine the quality of the fit. Any combination of values of $\vartheta_{k}$ and $p_{k}$ produce a surrogate that interpolates the sample points exactly. However, the values that provide the "best" kriging surrogate are obtained by maximizing the likelihood function, given in Eq. (5), which is a measure of the probability that the sample data was obtained from the assumed form of the SCF.

$$
-\frac{N_{s p} \ln \left(\sigma_{v a r}^{2}\right)+\ln \left|\mathbf{R}_{k r g}\right|}{2}
$$

The general procedure used to identify $\vartheta_{k}$ and $p_{k}$ is given in Ref. 22 .

As indicated in Refs. 20, 21, and 23, $f(\mathbf{x})=\beta$, where $\beta$ is constant, is often sufficient to produce approximations of sufficient accuracy. Therefore, in the current study it is assumed that $f(\mathbf{x})=\beta$. For this choice of $f(\mathbf{x})$, the generalized least-squares approximations of $\beta$ and $\sigma_{v a r}$ that are needed to obtain the kriging prediction are given by

$$
\hat{\beta}=\left(\mathbf{1}^{T}\left(\mathbf{R}_{k r g}\right)^{-1} \mathbf{1}\right)^{-1} \mathbf{1}^{T}\left(\mathbf{R}_{k r g}\right)^{-1} \mathbf{y}
$$

and

$$
\hat{\sigma}_{v a r}^{2}=\frac{(\mathbf{y}-\mathbf{1} \hat{\beta})^{T}\left(\mathbf{R}_{k r g}\right)^{-1}(\mathbf{y}-\mathbf{1} \hat{\beta})}{N_{s p}}
$$

The kriging prediction at a point $\mathbf{x}^{\text {new }}$ is given by

$$
\hat{y}_{k r g}\left(\mathbf{x}^{n e w}\right)=\hat{\beta}+\left(\mathbf{r}_{k r g}\left(\mathbf{x}^{n e w}\right)\right)^{T}\left(\mathbf{R}_{k r g}\right)^{-1}(\mathbf{y}-\mathbf{1} \hat{\beta})
$$

where

$$
\mathbf{r}_{k r g}\left(\mathbf{x}^{\text {new }}\right)=\left[\mathbf{R}_{k r g}\left(\mathbf{x}^{\text {new }}, \mathbf{x}^{1}\right), \ldots, \mathbf{R}_{k r g}\left(\mathbf{x}^{\text {new }}, \mathbf{x}^{N_{s p}}\right)\right]
$$

The accuracy of the surrogates is improved by adding new points to the set of existing fitting points. Two approaches are used to determine the new points. In the first approach, additional fitting points are determined in an ad hoc manner using an OLH sampling for specific regions of the parameter space where the accuracy of the surrogate is found to be inadequate.

In the second approach, the accuracy of the surrogates is improved in a systematic manner by using an efficient global optimization (EGO) algorithm. ${ }^{20,24,25}$ An expected improvement function (EIF) ${ }^{24,25}$, defined based on the local error in prediction, is used to identify regions of the parameter space where there is a high probability of producing a superior design over the current best design, and/or where the predictions of the surrogate are unreliable due to a high amount of uncertainty. Subsequently, additional sample points 
are identified by maximizing the EIF. An approximation of the local mean-squared error (MSE), obtained from kriging, is given by

$$
s^{2}(\mathbf{x})=\hat{\sigma}_{v a r}^{2}\left[1-\mathbf{r}_{k r g}^{T} \mathbf{R}_{k r g}^{-1} \mathbf{r}_{k r g}+\frac{1-\mathbf{1}^{T} \mathbf{R}_{k r g}^{-1} \mathbf{r}_{k r g}}{\mathbf{1}^{T} \mathbf{R}_{k r g}^{-1} \mathbf{1}}\right]
$$

Two types of EIFs can be defined

$$
E I F_{1}=\left(y_{m i n}-\hat{y}_{k r g}\right) \Phi_{d i s t}\left(\frac{y_{m i n}-\hat{y}_{k r g}}{s}\right)
$$

and

$$
E I F_{2}=s \phi_{\text {den }}\left(\frac{y_{\min }-\hat{y}_{k r g}}{s}\right)
$$

where the functions $\Phi_{d i s t}(*)$ and $\phi_{\text {den }}(*)$ represent the standard normal distribution function and standard normal density function, respectively. The function $E I F_{1}$ is large where $\hat{y}_{k r g}$ is likely to be better than $y_{\min }$. The term $E I F_{2}$ is large where the error metric $s(\mathbf{x})$ is large and thus where there is much uncertainty in the surrogate's prediction. A weighted expected improvement function (WEIF), given in Eq. (13), is used to control the search process in regions of the design space where there is considerable uncertainty in surrogate prediction and location of designs that are predicted to be optimal.

$$
W E I F(\mathbf{x})= \begin{cases}w E I F_{1}+(1-w) E I F_{2} & \text { if } s>0 \\ 0 & \text { if } s=0\end{cases}
$$

where $0 \leq w \leq 1$. Setting $w=0$ places more emphasis on searching in regions of the design space where there is significant uncertainty in the surrogate's predictions, whereas $w=1$ emphasizes locating designs that are predicted to be optimal.

\section{B. Optimization}

Once surrogates of acceptable accuracy are obtained, they are coupled to a suitable search algorithm to identify the set of design parameters that produce the desired combination of aerodynamic force and power. In the current study, a hybrid algorithm that employs global and gradient-based searches in sequence is used. The optimum predicted by the global approach is used as the initial guess for the gradient based approach. This allows for a somewhat less expensive search using the global approach. The design point predicted by the global search is expected to be a sufficiently good starting guess for the gradient based approach to identify the global optimum.

\section{Results and Discussion}

Results obtained from surrogate based optimization of rigid and flexible wings in hover are presented in this section. The results are obtained for Zimmerman wings, shown in Fig. 2(a), that have $R_{\text {span }}=75 \mathrm{~mm}$, $c_{r}=25 \mathrm{~mm}$, and an aspect ratio of 7.65 . The computations were carried out for air $\left(\rho_{\infty}=1.209 \mathrm{Kg} / \mathrm{m}^{3}\right.$ and $\nu_{\infty}=1.64 \times 10^{-5} \mathrm{~m}^{2} / \mathrm{s}$ ). Simulations using the approximate aerodynamic model were performed by dividing the wing into 10 spanwise stations, discretizing each airfoil using 101 elements, using 4 wake subiterations per time step, and a vortex core radius equal to $2.5 \%$ of the airfoil chord. The flow is assumed to be separated from the leading edge throughout the flapping stroke and the unsteady aerodynamic loads were computed using the unsteady Bernoulli equation. At the start of the motion, $X_{w}$ and $Y_{w}$, shown in Fig. 2(a), coincide with $X_{S P}$ and $Z_{S P}$, shown in Fig. 2(b), respectively. The thrust is defined as the component of the aerodynamic force resolved along $Z_{S P}$ shown in Fig. 2(b). The aerodynamic power is given by

$$
P_{\text {aero }}(t)=\iint_{\mathrm{A}_{\mathrm{w}}} \dot{\boldsymbol{x}}_{w}(t) \times \hat{\boldsymbol{n}}_{w} p \mathrm{dA}_{\mathrm{w}}
$$

In all simulations, an approximate steady state was obtained after one cycle. Therefore, the time averaged (or mean) quantities were calculated by averaging the corresponding time histories over cycles 2 through 5 . 


\section{A. Results for Rigid Wings}

The wings are actuated using a combined flap-pitch motion described by 5 parameters. The flapping and pitching motions, given in Eq. (15), are based on the kinematics considered in Ref. 8 and are prescribed about the flapping and pitching axes shown in Fig. 2(a).

$$
\Phi(t)=\Phi_{1} \frac{\sin ^{-1}\left[K_{\Phi} \sin (2 \pi f t)\right]}{\sin ^{-1}\left(K_{\Phi}\right)} \text { and } \alpha(t)=-\alpha_{1} \frac{\tanh \left[K_{\alpha} \sin \left(2 \pi f t+\varphi_{\alpha 1}\right)\right]}{\tanh \left(K_{\alpha}\right)}
$$

The kinematic parameters (design variables) and their allowed ranges are listed in the Table 1 . The parameters $K_{\Phi}$ and $K_{\alpha}$ govern the shape of the flap and pitch kinematic patterns as shown in Figs. 5.
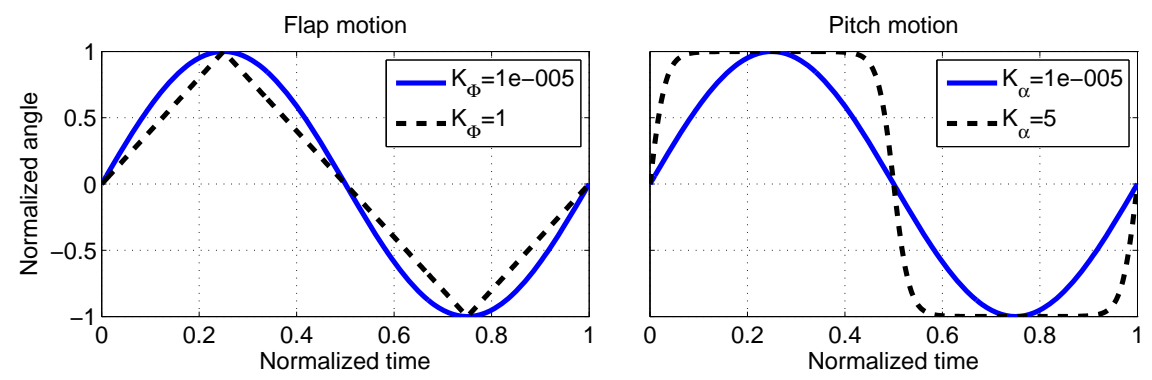

Figure 5. Variation of flap and pitch kinematic patterns with shape parameters

Table 1. Parameters and their allowed ranges

\begin{tabular}{ll}
\hline \hline Description & Range \\
\hline Flap amplitude & $10^{\circ} \leq \Phi_{1} \leq 60^{\circ}$ \\
Pitch amplitude & $0^{\circ} \leq \alpha_{1} \leq 60^{\circ}$ \\
Phase amplitude & $0^{\circ} \leq \phi_{\alpha 1} \leq 360^{\circ}$ \\
Shape parameter in flap & $0 \leq K_{\Phi} \leq 1$ \\
Shape parameter in pitch & $0 \leq K_{\alpha} \leq 5$ \\
Location of pitch axis & $0 \leq a \leq 0.25 c_{r}$ \\
Flapping frequency, $\mathrm{Hz}$ & $10 \leq f \leq 30$ \\
\hline
\end{tabular}

In previous studies using the aerodynamic model, ${ }^{11}$ simulations were conducted using 59 spanwise stations. In the current paper, only 10 spanwise stations were used in order to reduce the computational effort required to generate the fitting and testing data. The predictions using 10 sections were compared to those obtained using 59 spanwise stations at 50 points that were obtained from an OLH sampling of the parameter space using the iSight software. ${ }^{26,27}$ The maximum local errors in thrust and power are $6 \%$ and $3 \%$ of the corresponding maximum values. The root mean squared (RMS) errors in thrust and power are less than $2 \%$ of the corresponding maximum values. This implies that sufficient precision is achieved when using 10 spanwise stations.

Kriging surrogates to predict the mean thrust and mean power generated by rigid wings were created initially from a set of 200 points using the DACE toolbox in MATLAB ${ }^{\circledR} .{ }^{22}$ The accuracy of the surrogates was tested at the set of $50 \mathrm{OLH}$ points that were obtained previously. Each simulation needed to generate the fitting and testing data was conducted using $102.53 \mathrm{GHz}$ Intel Xeon E5540 processors (2GB RAM per processor). The maximum time per simulation was 1.5 hours. The mean and maximum errors in thrust and power are defined as follows:

$$
e_{j}=\frac{\left|\hat{y}_{k r g}^{j}-y^{j}\right|}{\max \left\{y^{j}\right\}} \quad \text { and } \quad e_{\text {max }}=\max \left\{e_{j}\right\} \quad \text { and } \quad e_{\text {mean }}=\frac{1}{N_{\text {test }}} \sum_{j=1}^{N_{\text {test }}} e_{j}
$$

Where $y_{k r g}^{j}$ and $y^{j}$ are the surrogate prediction and true function evaluation at the $j^{t h}$ test point. Next, the accuracy of the surrogates was improved by adding 149 new fitting points that were identified as follows. 
First, the initial surrogate was used in the EGO algorithm to identify 50 points. The surrogate was refitted by incorporating the new fitting points, and used in EGO a second time to identify 50 new points. The process was repeated a third time to identify 49 new points. The errors of the surrogates are summarized in Tables 2 and 3. Large errors were found to occur at the boundaries corresponding to $\alpha_{1}=0^{\circ}$, and $\left\{\Phi_{1}=60^{\circ}, f=30 \mathrm{~Hz}\right\}$. Therefore, an additional 400 points were generated using OLH samplings centered at these boundaries as follows. In the first sampling, 100 points were generated for $\alpha_{1}=0^{\circ}, 10^{\circ} \leq \Phi_{1} \leq 62.5^{\circ}$ and $10 \mathrm{~Hz}<f<32.5 \mathrm{~Hz}$, where the ranges for other parameters are given in Table 1 . In the second sampling, 300 points were generated for $52.5^{\circ} \leq \Phi_{1} \leq 62.5^{\circ}$ and $27.5 \mathrm{~Hz}<f<32.5 \mathrm{~Hz}$, where the ranges for other parameters are given in Table 1. This produced a significant reduction in the maximum error in thrust, as evident from Table 2(a). The reduction of maximum error in power was comparatively smaller, shown in Table 3(a). The distribution of errors are provided in Tables 2(b) and 3(b). The results in Tables 2 and 3 indicate that the surrogates have acceptable accuracy (errors $<20 \%$ ) over a large region of the parameter space.

Table 2. Errors in thrust

\begin{tabular}{cccc}
\hline \hline Errors & 197 points & 346 points & 738 points \\
\hline Mean & $6.3 \%$ & $5.9 \%$ & $4.2 \%$ \\
Max. & $52 \%$ & $72 \%$ & $20.6 \%$ \\
\hline
\end{tabular}

(a) Mean and maximum errors

\begin{tabular}{cccc}
\hline \hline Errors & 197 points & 346 points & 738 points \\
\hline$>20 \%$ & 4 points & 1 point & 1 point \\
$<20 \%$ & 46 points & 49 points & 49 points \\
\hline
\end{tabular}

(b) Distribution of errors

Table 3. Errors in power

\begin{tabular}{cccc}
\hline \hline Errors & 197 points & 346 points & 738 points \\
\hline Mean & $3.5 \%$ & $2.4 \%$ & $1.8 \%$ \\
Max. & $38.7 \%$ & $27 \%$ & $15.8 \%$ \\
\hline
\end{tabular}

(a) Mean and maximum errors

\begin{tabular}{cccc}
\hline \hline Errors & 197 points & 346 points & 738 points \\
\hline$>20 \%$ & 1 point & 1 point & 0 points \\
$<20 \%$ & 49 points & 49 points & 50 points \\
\hline
\end{tabular}

(b) Distribution of errors

The surrogates are combined with a hybrid search algorithm in order to identify kinematics that maximized thrust. The hybrid approach uses a global search based on the GODLIKE algorithm and a gradientbased search in sequence. The GODLIKE algorithm utilizes several global search approaches, such as genetic algorithms, simulated annealing, particle swarm, and differential evolution, to increase robustness of the optimization and is implemented in MATLAB ${ }^{\circledR}$ using the freely available GODLIKE toolbox. The gradient based approach uses an interior-point search and is implemented in MATLAB ${ }^{\circledR}$ using the fmincon function. The kinematic parameters corresponding to the maximum thrust, and predictions based on the surrogate and the true function evaluations are listed in Table 4 . The relative errors in thrust and power prediction are $3 \%$ and $8 \%$, respectively.

The variation of the thrust and power with the various parameters, calculated at the kinematics that produce maximum thrust, are shown in Figs. 6. The value of the kinematic parameter corresponding to maximum thrust is indicated by a circle. Positive thrust and power imply positive payload capacity and a power penalty, respectively.

Both the thrust and power increase monotonically with increase in flap amplitude, as shown in Fig. 6(a). This implies that thrust and power are competing design objectives. Figure 6(b) indicates that thrust and power are non-monotonic with pitch amplitude, suggesting that only a limited amount of pitching motion is useful. Both thrust and power vary significantly over the range of phase angles considered, as shown in Fig. 6(c). This implies that the phase difference between the flap and pitch motions can be used to tailor the thrust and power of a flapping wing without significant modification of the stroke amplitudes or flapping frequency. The effect of the shape parameters on the thrust and power are shown in Figs. 6(d) and 6(e). Figure 6(d) indicates that both thrust and power are non-monotonic as the flap motion changes from a sine motion to a triangular wave. A limited amount of triangular motion increases both thrust and power. The 

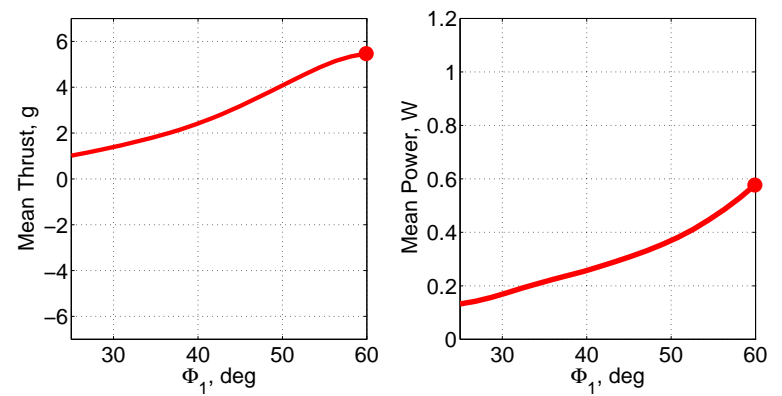

(a) Flap amplitude
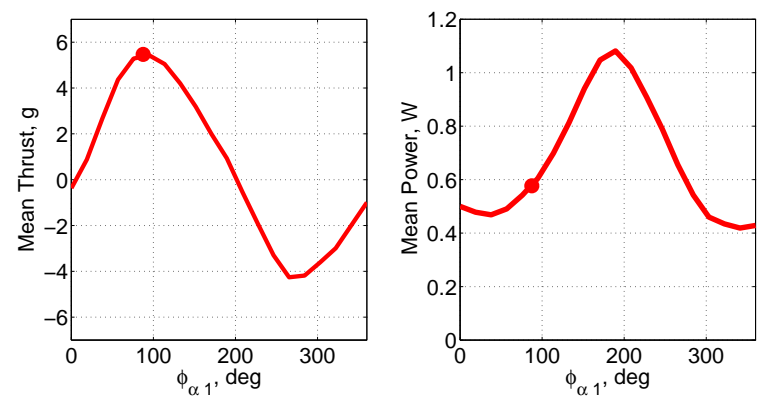

(c) Phase angle
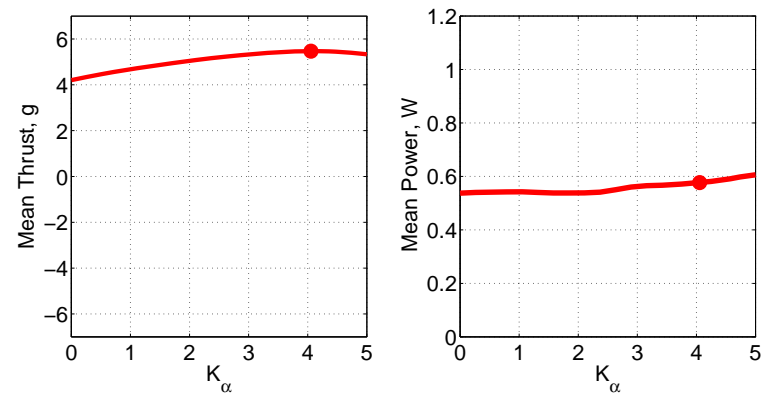

(e) Shape parameter for pitch motion
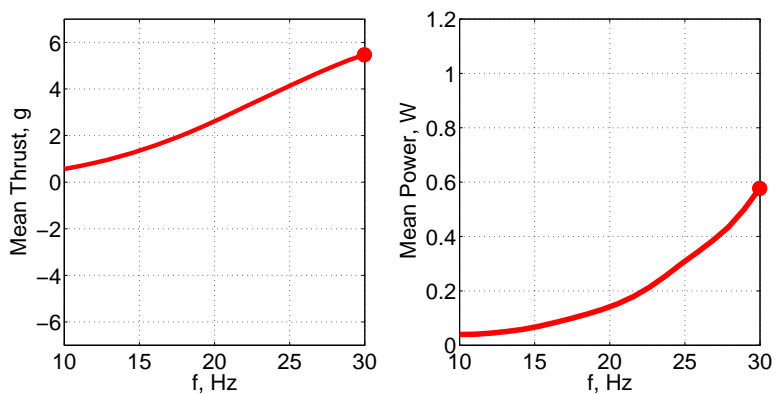

(g) Flapping frequency
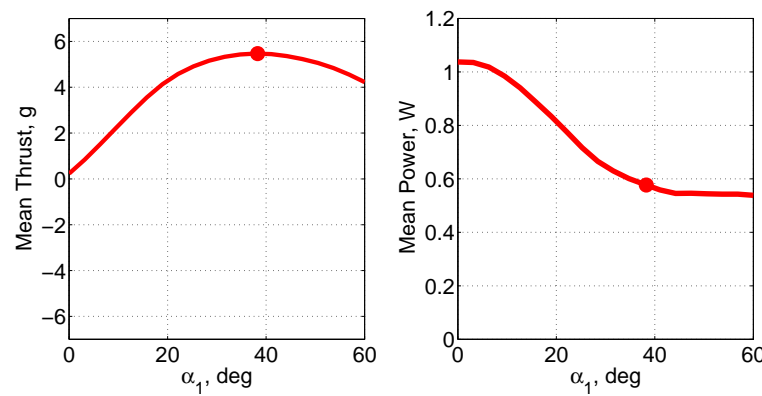

(b) Pitch amplitude
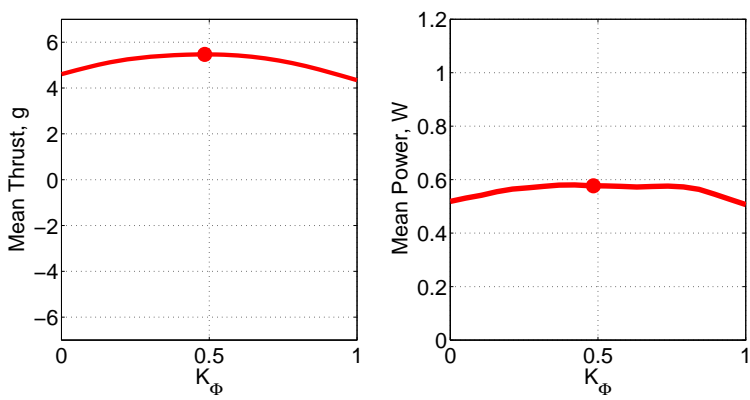

(d) Shape parameter for flap motion
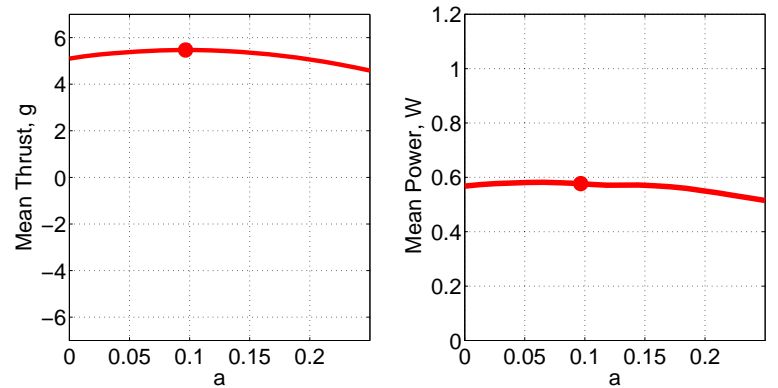

(f) Feathering axis offset 
Table 4. Design point corresponding to maximum thrust

\begin{tabular}{lccccccc}
\hline \hline Parameter & $\Phi_{1}$ & $\alpha_{1}$ & $\phi_{\alpha 1}$ & $K_{\Phi}$ & $K_{\alpha}$ & $a$ & $f$ \\
Value & $60^{\circ}$ & $39.37^{\circ}$ & $86.7^{\circ}$ & 0.47 & 4.14 & 0.095 & $30 \mathrm{~Hz}$ \\
\hline
\end{tabular}

(a) Kinematic parameters

\begin{tabular}{lcc}
\hline \hline & Surrogate prediction & True function evaluation \\
\hline$T_{\max }$ & $5.47 \mathrm{~g}$ & $5.31 \mathrm{~g}$ \\
$P_{T_{\max }}$ & $0.573 \mathrm{~W}$ & $0.527 \mathrm{~W}$ \\
\hline
\end{tabular}

(b) Surrogate predictions and true function evaluations

variations of thrust and power for the range of $K_{\Phi}$ considered are less than $20 \%$. Both thrust and power increase when a square wave is used for the pitching motion, as shown in Fig. 6(e). The increase in thrust is larger $(35 \%)$ compared to the increase in power $(<20 \%)$. This implies that a rapid pitch motion can be used to produce a significant increase in thrust with only a minor penalty on the power consumption. Figure $6(\mathrm{f})$ indicates that the power decreases by $15 \%$ as the location of the pitching axis moves from the leading edge $(a=0)$ to the quarter chord point $(a=0.25)$. The change in thrust is only $6 \%$. This suggests that pitching about the quarter chord is beneficial in reducing the power consumption with a comparatively minor reduction in thrust. Both thrust and power increase monotonically with increase in flapping frequency, as shown in Fig. 6(g).

A practical constraint in realistic vehicles is often the limitation of available power. Therefore, a constrained optimization study was conducted in which the thrust was maximized for a specified available power. The available power was varied from the power required to generate maximum thrust, denoted by $P_{T_{\max }}$, to $0.5 P_{T_{\max }}$. The pareto front, which represents the maximum thrust corresponding to the specified value of power, is shown in Fig. 7(a). It is interesting to see that the decrease in thrust is less than $20 \%$ of $T_{\max }$ for a $50 \%$ decrease in power. The true function evaluations at several points on the curve are represented by the red squares in Fig. 7(a). The relative errors between the surrogate predictions and the true function evaluations, plotted against the normalized value of the corresponding true function evaluation $\left(T / T_{\max }\right.$ or $\left.P / P_{T_{\max }}\right)$, are shown in Fig. $7(\mathrm{~b})$. The errors in thrust and power prediction, which are less than $15 \%$, are consistent with the errors listed in Tables 2 and 3.

\section{B. Results for Flexible Wings}

An aeroelastic optimization study was conducted using anisotropic wings built from an unstressed CAPRAN film (membrane) supported by a carbon fiber based spar-batten skeleton. The wing configuration is shown in Fig. 2(a). In the current study, the thicknesses of the leading edge spar and the five battens, shown in Fig. 2(a), are used as the design variables to govern flexibility. The material properties of the membrane and composite skeleton, the widths of the leading edge spar and the battens, and the thickness of the membrane are fixed ${ }^{11}$ and are provided in Table 5. The kinematic and structural parameters, which represent the complete set of design variables for the flexible wings, and their allowed ranges are given in Table 6 .

The finite element models of the wings, shown in Fig. 2(a), are composed of 765 shell elements (Element type 75 in MARC). Wing kinematics are implemented as displacement boundary conditions at the nodes that form the triangular root. The EOM obtained using the UL method are integrated forward in time using a single step Houbolt numerical scheme. ${ }^{28}$ During the simulations a pressure based filter, Ref. 16 (Ch. 7), described by Eq. (17) was used to limit the magnitude of numerical noise that is transmitted to the flexible wing.

$$
p_{\text {applied }}= \begin{cases}-p_{\text {limit }} & \text { if } \quad p_{\text {calculated }} \leq-p_{\text {limit }} \\ p_{\text {calculated }} & \text { if }-p_{\text {limit }} \leq p_{\text {calculated }} \leq p_{\text {limit }} \\ p_{\text {limit }} & \text { if } p_{\text {calculated }} \geq p_{\text {limit }}\end{cases}
$$




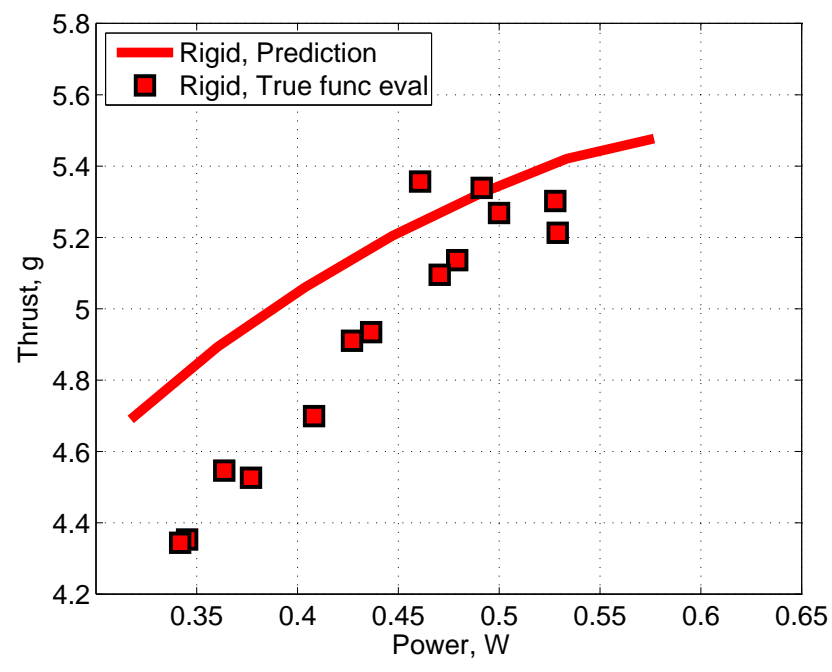

(a) Maximum thrust for specified power

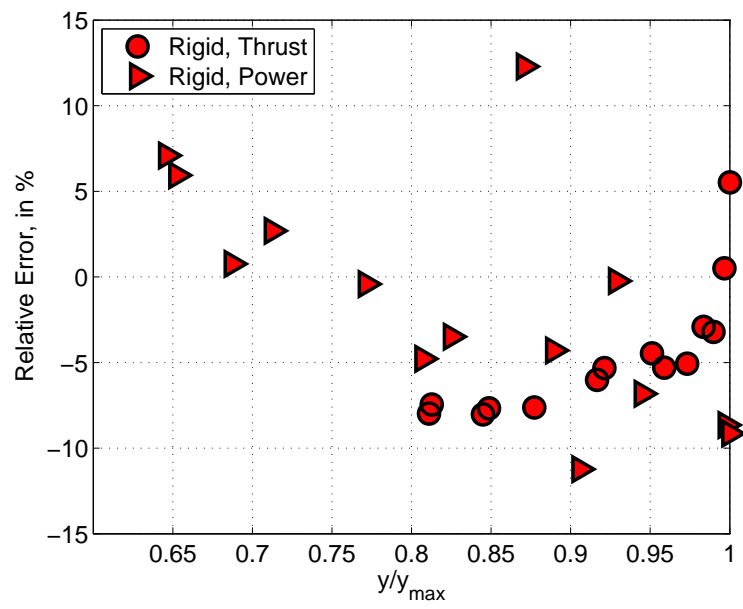

(b) Errors in prediction of thrust and power

Figure 7. Constrained optimization of rigid wings

For the cases considered, a limiting pressure given by $p_{\text {limit }}=81 p_{r e f}$, where $p_{r e f}$ as defined in Eq. (18), was used.

$$
p_{\text {ref }}=\frac{1}{2} \rho_{\infty} U_{\text {tip }}^{2} \quad \text { where } \quad U_{\text {tip }}=2 \pi f \beta_{0} R_{\text {span }}
$$

Table 5. Material properties of the composite skeleton and membrane

\begin{tabular}{cc}
\hline \hline$E_{11}$ & $233 \mathrm{GPa}$ \\
$E_{22}$ & $23.1 \mathrm{GPa}$ \\
$E_{12}$ & $10.5 \mathrm{GPa}$ \\
$\nu_{12}$ & 0.05 \\
$\rho$ & $1740 \mathrm{~kg} / \mathrm{m}^{3}$ \\
\hline
\end{tabular}

(a) Composite

\begin{tabular}{cc}
\hline \hline$E$ & $2.76 \mathrm{GPa}$ \\
$\nu_{12}$ & 0.489 (Incompressible) \\
$\rho$ & $1384 \mathrm{~kg} / \mathrm{m}^{3}$ \\
Thickness & $15 \mathrm{microns}$ \\
\hline
\end{tabular}

(b) Membrane

The kriging surrogates were created initially from a set of 199 points that were generated using an OLH sampling of the parameter space described in Table 6 . The accuracy of the surrogates was tested at the set of 49 points, which were also generated by an OLH sampling of the parameter space described in Table 6 . Each simulation needed to generate the fitting and testing data was conducted using a single $2.53 \mathrm{GHz}$ Intel Xeon E5540 processor (4GB RAM). The maximum time per simulation was 15 hours. The mean and maximum errors in thrust and power are provided in Tables 8 and 9, respectively. The accuracy of the surrogates was first improved by adding 372 new fitting points that were identified using EGO. These points were determined in 4 cycles, where the surrogate was used in EGO and re-fitted by including the new points for the next cycle. The surrogates were found to be reasonably accurate over a significant portion of the domain. Large errors were found to occur at the boundary corresponding to $\left\{\Phi_{1}=55^{\circ}, f=25 \mathrm{~Hz}\right\}$. Therefore, an OLH sampling centered at this boundary was considered. An additional 276 points were generated for the ranges given in Table 7 . This decreased the mean and maximum errors in power, and the maximum error in thrust. However, the mean error in thrust was found to increase slightly. The magnitude and distribution of errors of the surrogates are summarized in Tables 8 and 9 . These results indicate that the surrogates have acceptable accuracy (errors $<20 \%$ ) over a large region of the parameter space.

The kinematic and structural parameters that produce maximum thrust are given in Table 10. The 
Table 6. Parameters and their allowed ranges

\begin{tabular}{ll}
\hline \hline Description & Range \\
\hline Flap amplitude & $10^{\circ} \leq \Phi_{1} \leq 55^{\circ}$ \\
Pitch amplitude & $0^{\circ} \leq \alpha_{1} \leq 50^{\circ}$ \\
Phase amplitude & $0^{\circ} \leq \phi_{\alpha 1} \leq 360^{\circ}$ \\
Shape parameter in flap & $0 \leq K_{\Phi} \leq 0.8$ \\
Shape parameter in pitch & $0 \leq K_{\alpha} \leq 4$ \\
Location of pitch axis & $0 \leq a \leq 0.25 c_{r}$ \\
Flapping frequency, Hz & $10 \leq f \leq 25$ \\
Thickness of LE spar, mm & $0.15 \leq t_{l e ~ s p a r} \leq 0.4$ \\
Thickness of Rib 1, mm & $0.2 \leq t_{r i b 1} \leq 0.3$ \\
Thickness of Rib 2, mm & $0.05 \leq t_{r i b 2} \leq 0.3$ \\
Thickness of Rib 3, mm & $0.05 \leq t_{\text {rib3 }} \leq 0.3$ \\
Thickness of Rib $4, \mathrm{~mm}$ & $0.05 \leq t_{r i b 4} \leq 0.3$ \\
Thickness of Rib $5, \mathrm{~mm}$ & $0.05 \leq t_{r i b 5} \leq 0.3$ \\
\hline
\end{tabular}

Table 7. Reduced ranges for the parameters

\begin{tabular}{ll}
\hline \hline Description & Range \\
\hline Flap amplitude & $52.5^{\circ} \leq \Phi_{1} \leq 57.5^{\circ}$ \\
Pitch amplitude & range given in Table 6 \\
Phase amplitude & range given in Table 6 \\
Shape parameter in flap & range given in Table 6 \\
Shape parameter in pitch & range given in Table 6 \\
Location of pitch axis & range given in Table 6 \\
Flapping frequency, Hz & $22.5 \leq f \leq 27.5$ \\
Thickness of LE spar, mm & range given in Table 6 \\
Thickness of Rib 1, mm & $0.05 \leq t_{r i b 1} \leq 0.3$ \\
Thickness of Rib 2, mm & $0.025 \leq t_{r i b 2} \leq 0.3$ \\
Thickness of Rib 3, mm & $0.025 \leq t_{r i b 3} \leq 0.3$ \\
Thickness of Rib 4, mm & $0.025 \leq t_{r i b 4} \leq 0.3$ \\
Thickness of Rib $5, \mathrm{~mm}$ & $0.025 \leq t_{r i b 5} \leq 0.3$ \\
\hline
\end{tabular}

corresponding parameters obtained for rigid wings are also provided. The true function evaluations for thrust yields $3.25 \mathrm{~g}$ for rigid wings and $3.73 \mathrm{~g}$ for flexible wings. The corresponding evaluations for power yield $0.249 \mathrm{~W}$ and $0.256 \mathrm{~W}$ for rigid and flexible wings, respectively. This indicates that the best flexible configuration generates 15\% more thrust and consumes 3\% more power compared to the best rigid wing.

The variation of thrust and power with kinematic and structural parameters, calculated at the point of maximum thrust, are shown in Figs. 8 and 9, respectively. Several similarities to the variation of thrust and power obtained for rigid wings (shown using the dashed red line) are noted. Both the thrust and power increase monotonically with increase in flap amplitude, as shown in Fig. 8(a). Figure 8(b) indicates that thrust and power are non-monotonic with pitch amplitude, suggesting that only a limited amount of pitching motion is useful. The best flexible configuration generates greater thrust compared to rigid wings while requiring a smaller amplitude in pitching motion. This is because the flexible wings undergo twist thereby producing a pitch distribution. Both thrust and power vary significantly over the range of phase angles considered, as shown in Fig. 8(c). The variation of thrust with phase angle for flexible wings is similar to the corresponding variation for rigid wings. The variation of power is somewhat less significant. This suggests that the phase difference between flap and pitch motions for flexible wings can be used to tailor the thrust for fixed stroke amplitudes and frequency. 
Table 8. Errors in thrust

\begin{tabular}{cccc}
\hline \hline Errors & 199 points & 571 points & 847 points \\
\hline Mean & $10.5 \%$ & $4.5 \%$ & $4.7 \%$ \\
Max. & $43 \%$ & $19.3 \%$ & $15.3 \%$ \\
\hline
\end{tabular}

(a) Mean and maximum errors

\begin{tabular}{cccc}
\hline \hline Errors & 199 points & 571 points & 847 points \\
\hline$>20 \%$ & 6 points & 0 points & 0 points \\
$<20 \%$ & 43 points & 49 points & 49 points \\
\hline
\end{tabular}

(b) Distribution of errors

Table 9. Errors in power

\begin{tabular}{cccc}
\hline \hline Errors & 199 points & 571 points & 847 points \\
\hline Mean & $6.6 \%$ & $5.6 \%$ & $3 \%$ \\
Max. & $22 \%$ & $30 \%$ & $22 \%$ \\
\hline
\end{tabular}

(a) Mean and maximum errors

\begin{tabular}{cccc}
\hline \hline Errors & 199 points & 571 points & 847 points \\
\hline$>20 \%$ & 2 points & 1 point & 1 point \\
$<20 \%$ & 47 points & 48 points & 48 points \\
\hline
\end{tabular}

(b) Distribution of errors

The effect of the shape parameters on the thrust and power are shown in Figs. 8(d) and 8(e). Figure 8(d) indicates that the thrust increases slightly as the flap motion changes from a cosine motion to a triangular wave. The variation of power is minor compared to the reduction in thrust, implying that flap motion based on a triangular wave is beneficial. For flexible wings, the thrust and power show only a minor variation as $K_{\alpha}$ increases, as shown in Fig. 8(e). This implies that a rapid pitch motion at the end of the flapping stroke has a greater impact on rigid wing performance compared to flexible wings. Figure $8(\mathrm{f})$ shows the thrust increases slightly and power decreases slightly as the pitching axis moves from the leading edge of the wing to the quarter-chord point, implying that it is beneficial to pitch about the quarter-chord point. Note that opposite trend is obtained for thrust generated by rigid wings, wherein it is beneficial to pitch about a point closer to the leading edge.

Both thrust and power increase with increase in flapping frequency, as shown in Fig. 8(g). It is interesting to note that flexible wings generate more thrust compared to rigid wings only for $f>22 \mathrm{~Hz}$. Below $22 \mathrm{~Hz}$, rigid wings produce slightly greater thrust for equal or less required power compared to flexible wings. Therefore, increasing wing flexibility in this region of the parameter space would result in reduction of thrust and a possible increase in required power.

The variation of thrust and power with structural parameters are shown in Figs. 9. The thickness of the LE spar has a significant effect on both the thrust generated and power consumed by the flexible wings, as shown in Fig. 9(a). The variation of thrust with $t_{\text {le spar }}$ is non-monotonic suggesting that only a limited amount of spanwise stiffness is beneficial. Both thrust and power increase with increase in $t_{\text {le spar }}$, implying that increasing wing stiffness enhances thrust production, but also increases the power penalty. The variation of thrust and power with the thickness of ribs 1, 2, 3, and 4, shown in Figs. 9(b) to 9(e), is somewhat minor. Rib 5 affects the tip twist and therefore has moderate impact on the thrust, shown in Fig. 9(f).

Results of a constrained optimization, in which the available power was specified as a fraction of the $P_{T_{\max }}$ is shown in Fig. 10(a). The corresponding results obtained for rigid wings for the range of kinematic parameters listed in Table 6 are shown. The true function evaluations at several points on the curves are also shown. The 'best' flexible configuration predicted by the surrogates produces $7 \%$ more thrust and requires $5 \%$ more power compared to the 'best' rigid configuration. The true function evaluations show that the best flexible configuration produces $15 \%$ more thrust and requires $3 \%$ more power compared to the 'best' rigid configuration. The surrogates predict that flexible wings generate greater thrust compared to rigid wings for a given requirement of power. This trend is reflected in the true function evaluations, which indicate that flexible configurations produced equal or greater thrust compared to rigid configurations while requiring similar values of power. Thus, the surrogates adequately capture the trends in both thrust and power. Several flexible configurations produced equal or greater thrust compared to the best rigid configuration. However, they required similar or greater values of power. This suggests that the choice between rigid and flexible wings based on performance is not straightforward. 

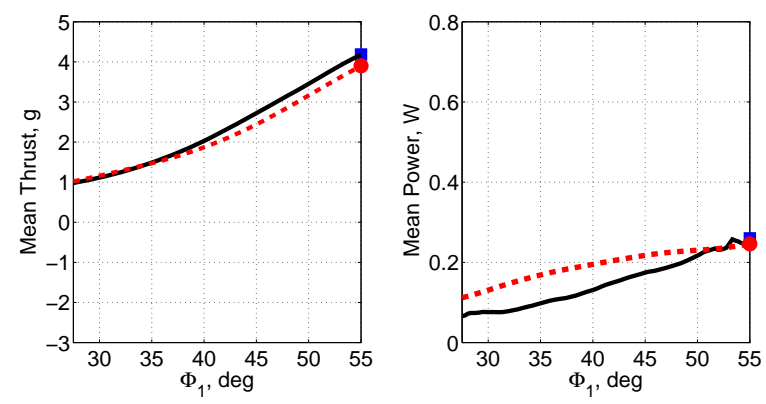

(a) Flap amplitude
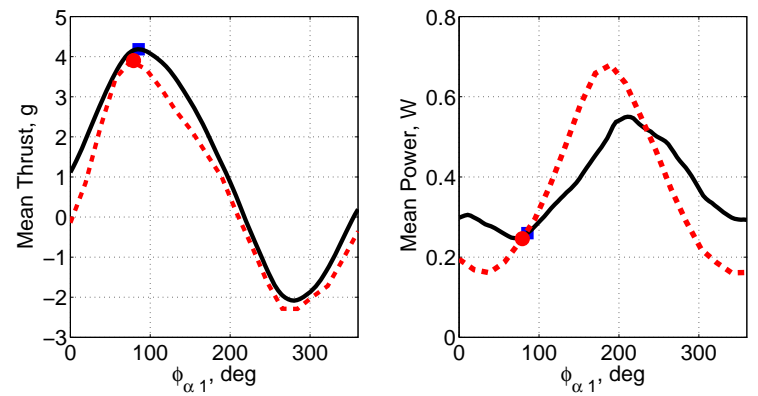

(c) Phase angle
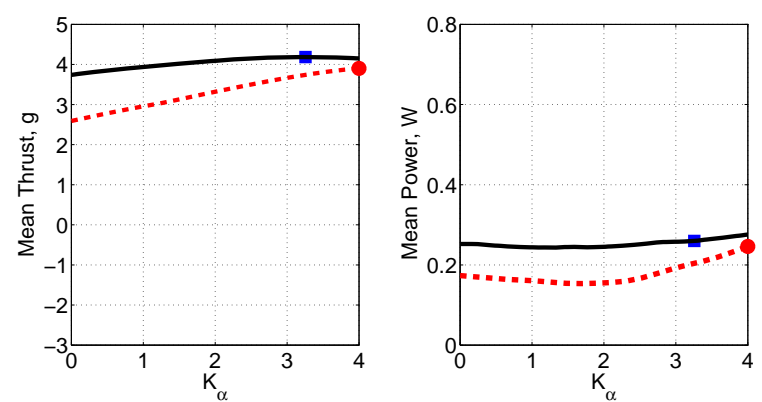

(e) Shape parameter for pitch motion
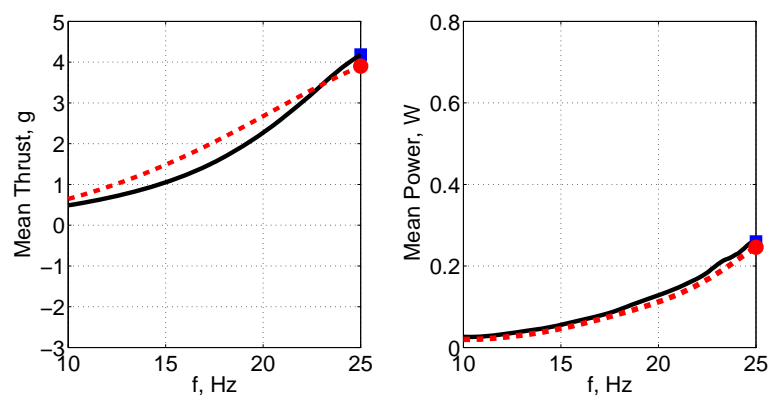

(g) Flapping frequency

Figure 8. Variation of thrust and power with kinematic parameters: Flexible wings - solid line, rigid wings dashed line

16 of 21

American Institute of Aeronautics and Astronautics 

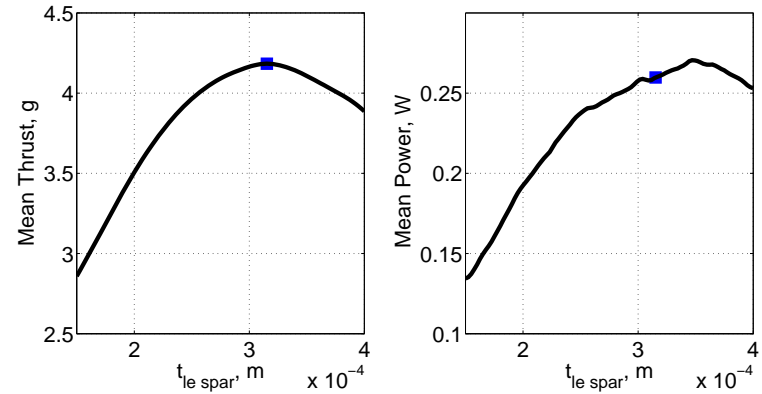

(a) Thickness of LE spar
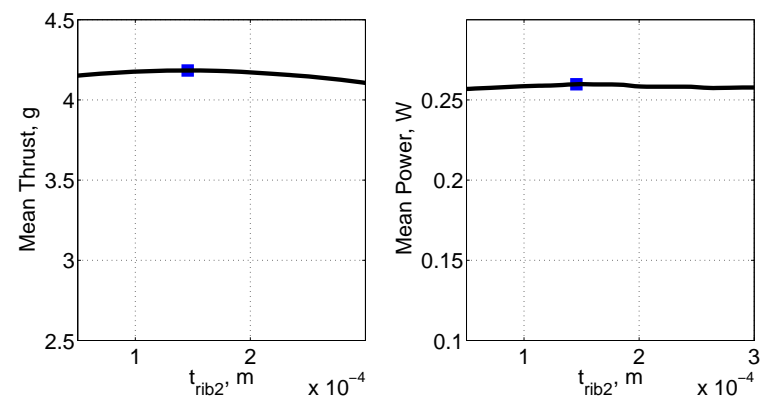

(c) Thickness of Rib 2
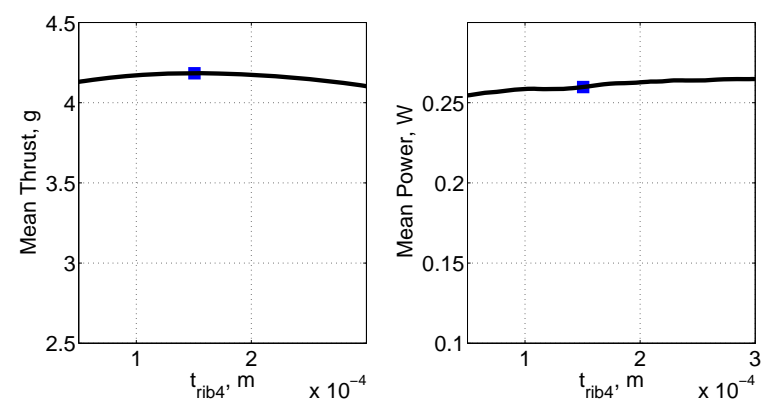

(e) Thickness of Rib 4
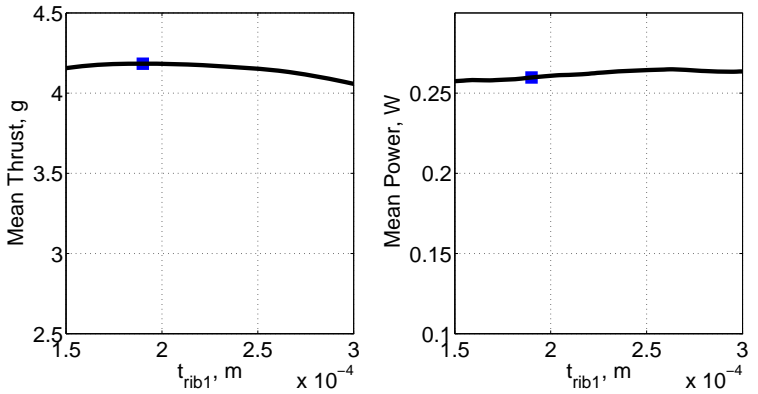

(b) Thickness of Rib 1
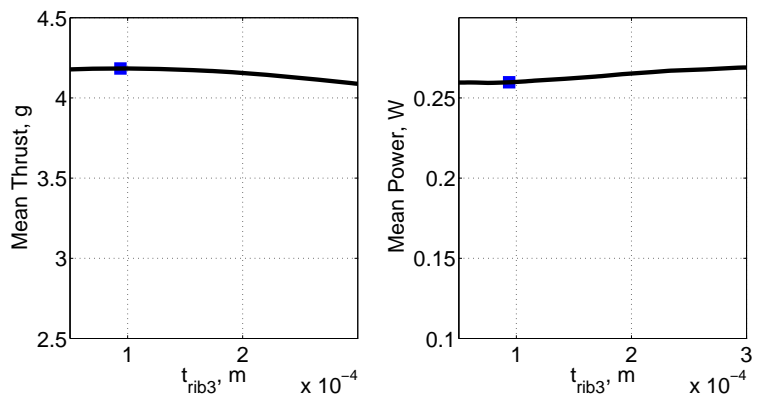

(d) Thickness of Rib 3
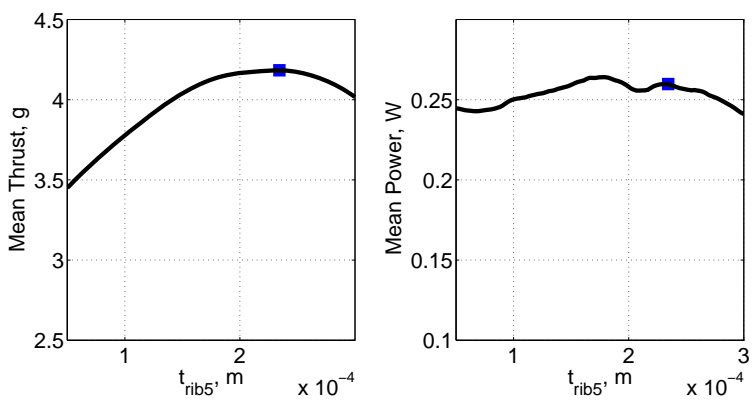

(f) Thickness of Rib 5

Figure 9. Variation of thrust and power with structural parameters 
Table 10. Kinematic and structural parameters corresponding to maximum thrust

\begin{tabular}{rcc}
\hline \hline Description & Rigid Wing & Flexible Wing \\
\hline$\Phi_{1}$ & $55^{\circ}$ & $55^{\circ}$ \\
$\alpha_{1}$ & $46.6^{\circ}$ & $37.6^{\circ}$ \\
$\phi_{\alpha 1}$ & $79.1^{\circ}$ & $85.5^{\circ}$ \\
$K_{\Phi}$ & 0.49 & 0.64 \\
$K_{\alpha}$ & 4 & 3.25 \\
$a$ & 0.51 & 0.18 \\
$f$ & $25 \mathrm{~Hz}$ & $25 \mathrm{~Hz}$ \\
\hline$t_{\text {le spar }}$ & & $0.3154 \mathrm{~mm}$ \\
$t_{\text {rib1 }}$ & & $0.19 \mathrm{~mm}$ \\
$t_{\text {rib } 2}$ & & $0.1455 \mathrm{~mm}$ \\
$t_{\text {rib } 3}$ & & $0.0937 \mathrm{~mm}$ \\
$t_{\text {rib4 }}$ & & $0.1505 \mathrm{~mm}$ \\
$t_{\text {rib } 5}$ & & $0.2343 \mathrm{~mm}$ \\
\hline$T_{\text {max }}$, prediction & $3.9 \mathrm{~g}$ & $4.18 \mathrm{~g}$ \\
True evaluation & $3.25 \mathrm{~g}$ & $3.73 \mathrm{~g}$ \\
$P_{\text {Tmax }}$, prediction & $0.247 \mathrm{~W}$ & $0.26 \mathrm{~W}$ \\
True evaluation & $0.249 \mathrm{~W}$ & $0.256 \mathrm{~W}$ \\
\hline
\end{tabular}

The relative errors in prediction, plotted against the normalized value of the corresponding true function evaluation $\left(T / T_{\max }\right.$ or $\left.P / P_{T_{\max }}\right)$, are shown in Fig. $10(\mathrm{~b})$. The surrogates for thrust have acceptable accuracy (errors $\leq 20 \%$ ). The errors in prediction of power are larger, with over- or under-prediction of up to $30 \%$. For both rigid and flexible wings, the errors in prediction of power increase as $P / P_{T \max }$ decreases, suggesting that the accuracy of the surrogates may need further improvement in regions of the parameter space where the power required by the configurations is lower than $0.8 P_{\operatorname{Tmax}}$. 


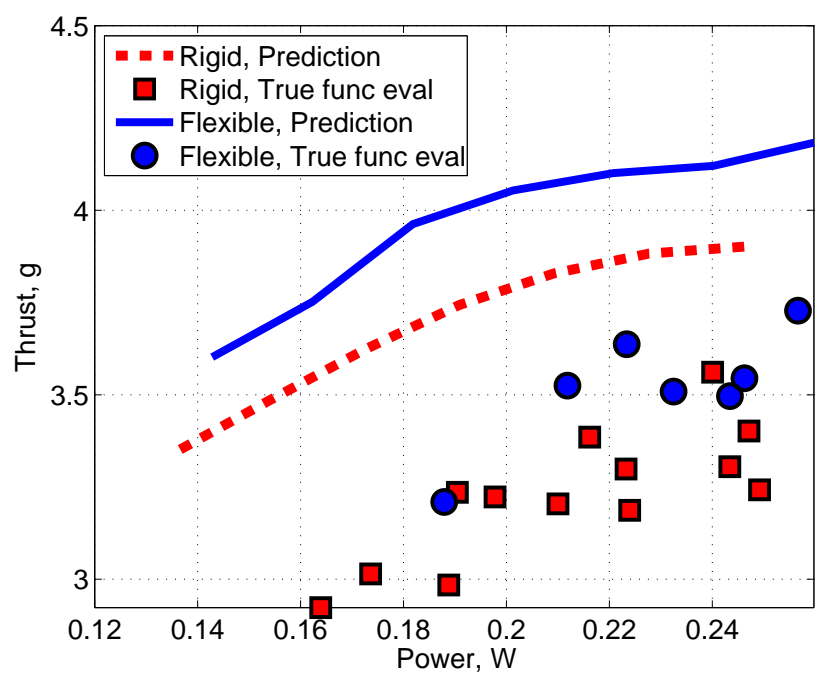

(a) Maximum thrust for specified power

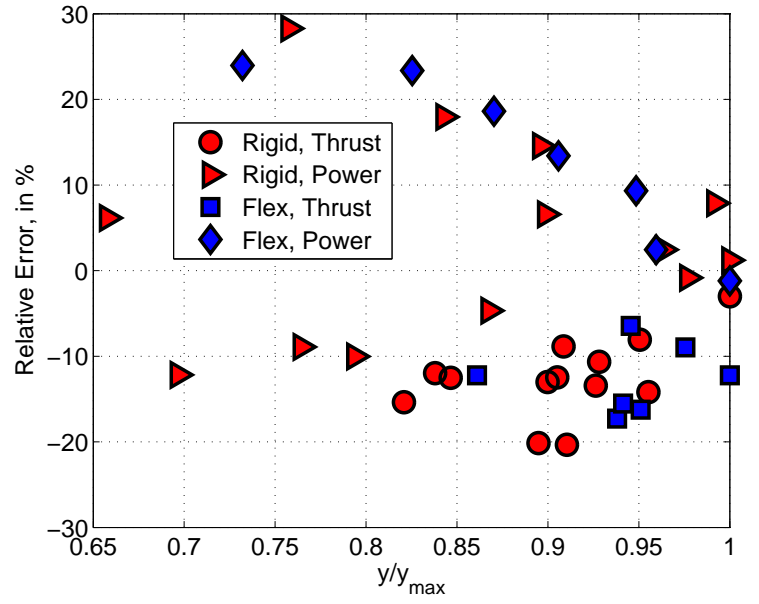

(b) Errors in prediction of thrust and power

Figure 10. Constrained optimization of rigid and flexible wings

\section{Concluding Remarks}

A surrogate-based optimization of rigid and flexible flapping wings in hover is described. The surrogates, generated using kriging from a set of fitting points, are used to predict the time-averaged thrust generated and the power required by the wings. The accuracy of the surrogates was improved using a combination of the EGO approach and using an OLH sampling centered at the boundaries. The surrogates were used to examine the variation of thrust and power with the kinematic and structural parameters. Constrained optimization studies, in which the thrust was maximized for a specified amount of available power, were also conducted. The following conclusions can be gleaned from the results.

1. The thrust and power are competing quantities for both rigid and flexible wings. Therefore, wing design and kinematic selection involves a trade-off between thrust generation and power consumption.

2. The surrogates, for both rigid and flexible wings, identified configurations that were better than the best configuration in the set of fitting points.

3. The rigid wings were found to offer similar, if not better, performance compared to flexible wings in several regions of the parameter space. Thus, the choice between rigid and flexible wings for a given level of performance is not straightforward.

4. A rapid pitching motion at the ends of the flapping stroke was beneficial for both rigid and flexible wings.

5. The phase angle between flap and pitch motions was found to have significant impact on the thrust generated and the power consumed by the wings. Thus, the phase angle can be used to tailor the performance of the wings while keeping the stroke amplitudes and flapping frequency fixed.

6. For flexible wings, the thickness of the leading edge spar had the greatest impact on the thrust generated and power consumed by the wings compared to the other structural parameters.

7. The peak performance of flexible wings was achieved using a pitch actuation that was smaller than the corresponding actuation required for rigid wings. This is because the wing twist produces an additional angle of attack distribution that generates the forces. This also implies that wing flexibility can be passively tailored to achieve peak performance while reducing the complexity in the actuation mechanism. 


\section{Acknowledgments}

This work was supported by the Air Force Office of Scientific Research's (AFOSR) MURI with Dr. Douglas R. Smith as Program Director.

\section{References}

${ }^{1}$ Mueller, T. J., Fixed and Flapping Wing Aerodynamics for Micro Air Vehicle Applications, Vol. 195, Progress in Aeronautics and Astronautics, published by AIAA, 2001.

${ }^{2}$ Sane, S. P., "The Aerodynamics of Insect Flight," The Journal of Experimental Biology, Vol. 206, 2003, pp. 4191 - 4208.

${ }^{3}$ Shyy, W., Lian, Y., Tang, J., Liu, H., Trizila, P., Stanford, B., Bernal, L., Cesnik, C., Friedmann, P., and Ifju, P., "Computational Aerodynamics of Low Reynolds Number Plunging, Pitching, ad Flexible Wings for MAV Applications," 48th AIAA Aerospace Sciences Meeting and Exhibit, Reno, Nevada, January 2008, pp. 1-33, AIAA Paper No. 2008-523.

${ }^{4}$ Shyy, W., Lian, Y., Tang, J., Viieru, D., and Liu, H., Aerodynamics of Low Reynolds Number Flyers, Cambridge University Press, 2008.

${ }^{5}$ Shyy, W., Aono, H., Chimakurthi, S., Trizila, P., Kang, C.-K., Cesnik, C., and Liu, H., "Recent Progress in Flapping Wing Aerodynamics and Aeroelasticity," Progress in Aerospace Sciences, Vol. 46, No. 7, 2010, pp. $284-327$.

${ }^{6}$ Aono, H., Liang, F., and Liu, H., "Near- and Far-Field Aerodynamics in Insect Hovering Flight: An Integrated Computational Study," The Journal of Experimental Biology, Vol. 211, No. 2, 2008, pp. 239-257.

${ }^{7}$ Keennon, M., Klingebiel, K., Won, H., and Andriukov, A., "Development of the Nano Hummingbird: A Tailless Flapping Wing Micro Air Vehicle," 50th AIA A Aerospace Sciences Meeting including the New Horizons Forum and Aerospace Exposition, Nashville, Tennessee, 9 - 12 January 2012, pp. 1 - 24, AIAA Paper 2012-0588.

${ }^{8}$ Berman, G. J. and Wang, Z. J., "Energy-Minimizing Kinematics in Hovering Insect Flight," Journal of Fluid Mechanics, Vol. 582, 2007, pp. 153-168.

${ }^{9}$ Tuncer, I. H. and Kaya, M., "Optimization of flapping airfoils for maximum thrust and propulsive efficiency," AIAA Journal, Vol. 43, No. 11, 2005, pp. 2329 - 2336.

${ }^{10}$ Stanford, B., Kurdi, M., Beran, P., and McClung, A., "Shape, Structure, and Kinematic Parameterization of a PowerOptimal Hovering Wing," 51st AIAA/ASME/ASCE/AHS/ASC Structures, Structural Dynamics, and Materials Conference, Orlando, Florida, 12-15 April 2010, pp. 1 - 18, AIAA Paper 2010-2963.

${ }^{11}$ Gogulapati, A., Friedmann, P. P., Kheng, E., and Shyy, W., "Approximate Aeroelastic Modeling of Flapping Wings in Hover," AIAA Journal, Vol. 51, No. 3, March 2013, pp. 1-17.

${ }^{12}$ Gogulapati, A. and Friedmann, P. P., "Approximate Aeroelastic Analysis of Flapping Wings in Hover and Forward Flight," 52nd AIAA/ASME/ASCE/AHS/ASC Structures, Structural Dynamics, and Materials Conference, Denver, Colorado, 4-7 April 2011, pp. 1-31, AIAA Paper 2011-2008.

${ }^{13}$ Queipo, N., Haftka, R., Shyy, W., Goel, T., Vaidyanathan, R., and Kevin Tucker, P., "Surrogate-based analysis and optimization," Progress in Aerospace Sciences, Vol. 41, No. 1, 2005, pp. 1-28.

${ }^{14}$ Trizila, P., Kang, C.-K., Visbal, M., and Shyy, W., "Unsteady Fluid Physics and Surrogate Modeling of Low Reynolds Number, Flapping Airfoils," 38th AIAA fluid dynamics conference and exhibit, Seattle, Washington, 23-26 June 2008, pp. 1-22, AIAA Paper 2008-3821.

${ }^{15}$ MSC. MARC, Volumes A - D, 2005.

${ }^{16}$ Gogulapati, A., Nonlinear Approximate Aeroelastic Analysis of Flapping Wings in Hover and Forward Flight, Ph.D. thesis, University of Michigan, Ann Arbor, 2011.

${ }^{17}$ Ansari, S. A., Żbikowski, R., and Knowles, K., "Non-linear Unsteady Aerodynamic Model for Insect-like Flapping Wing in the Hover. Part 1: Methodology and Analysis," Proceedings of the I MECH E Part G Journal of Aerospace Engineering, Vol. 220, No. 2, 2006, pp. 61-83.

${ }^{18}$ Ansari, S. A., Żbikowski, R., and Knowles, K., "Non-linear Unsteady Aerodynamic Model for Insect-like Flapping Wing in the Hover. Part 2: Implementation and Validation," Proceedings of the I MECH E Part G Journal of Aerospace Engineering, Vol. 220, No. 2, 2006, pp. 169-186.

${ }^{19}$ Bathe, K., Finite Element Procedures, Prentice-Hall Inc., 1996.

${ }^{20}$ Glaz, B., Friedmann, P., and Liu, L., "Helicopter Vibration Reduction Throughout the Entire Flight Envelope Using Surrogate-Based Optimization," Journal of the American Helicopter Society, Vol. 54, No. 1, 2009, pp. 12007-12007.

${ }^{21}$ Forrester, A. I. J., Sóbester, A., and Keane, A. J., Engineering Design Via Surrogate Modelling: A Practical Guide, Wiley, 2008.

${ }^{22}$ Lophaven, S. N., Nielsen, H. B., and Sondergaard, J., "A Matlab Kriging Toolbox, Version 2.0," Tech. Rep. Technical Report IMM-TR-2002-12, Informatics and Mathematical Modeling, DTU, 2002, Available at http://www2.imm.dtu.dk/ $\mathrm{hbn} /$ dace/.

${ }^{23}$ Simpson, T. W., Poplinski, J. D., Koch, P. N., and Allen, J. K., "Metamodels for Computer-Based Engineering Design: Survey and Recommendations," Engineering with Computers, Vol. 17, No. 2, 2001, pp. 129-150.

${ }^{24}$ Jones, D. R., Schonlau, M., and Welch, W. J., "Efficient Global Optimization of Expensive Black-Box Functions," Journal of Global Optimization, Vol. 13, No. 4, 1998, pp. 455-492.

${ }^{25}$ Jones, D. R., "A Taxonomy of Global Optimization Methods Based on Response Surfaces," Journal of Global Optimization, Vol. 21, No. 4, 2001, pp. 345-383.

${ }^{26}$ Jin, R., Chen, W., and Sudjianto, A., "An Efficient Algorithm for Constructing Optimal Design of Computer Experiments," Journal of Statistical Planning and Inference, Vol. 134, No. 1, 2005, pp. 268-287. 
${ }^{27}$ Koch, P. N., Evans, J. P., and Powell, D., "Interdigitation For Effective Design Space Exploration Using iSIGHT," Structural and Multidisciplinary Optimization, Vol. 23, No. 2, 2002, pp. 111-126.

${ }^{28}$ Chung, J. and Hulbert, G. M., "A Family of Single-Step Houbolt Time Integration Algorithms for Structural Dynamics," Computational Methods in Applied Mechanics and Engineering, Vol. 118, 1994, pp. 1 - 11. 